\title{
An unusual cause of massive upper gastrointestinal bleeding: Dieulafoy's lesion within a giant mid- esophageal diverticulum
}

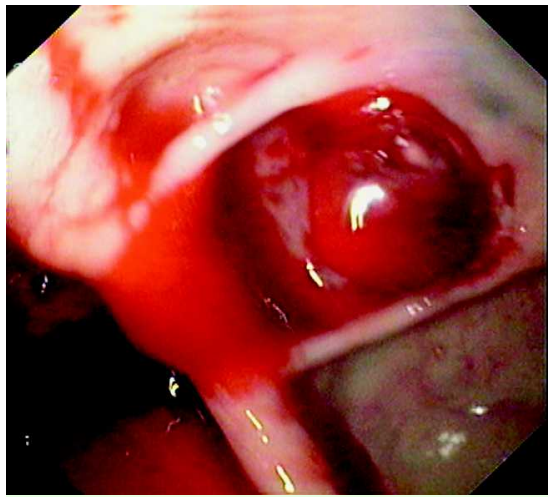

Fig. 1 Endoscopic view of Dieulafoy's lesion with oozing blood within the diverticulum.

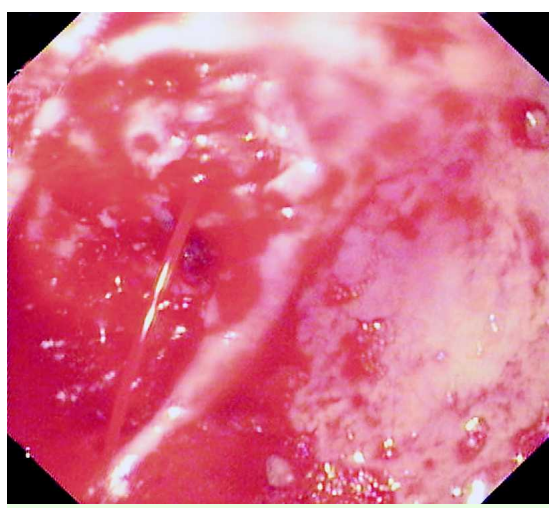

Fig. 2 Endoscopic view of actively spurting Dieulafoy's lesion within the diverticulum.

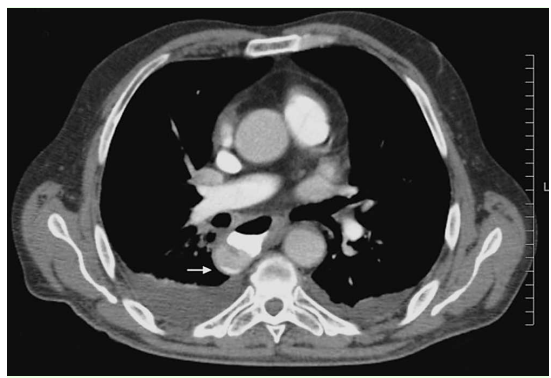

Fig. 3 Thorax computed tomography scan with an oral contrast medium demonstrates huge esophageal diverticulum (white arrow).

Midesophageal diverticula are usually asymptomatic and are often discovered incidentally during endoscopy performed for unrelated reasons. A small proportion present with life-threatening bleeding. We present here a first report of the massive arterial bleeding from Dieulafoy's lesion in a midesophageal diverticulum.

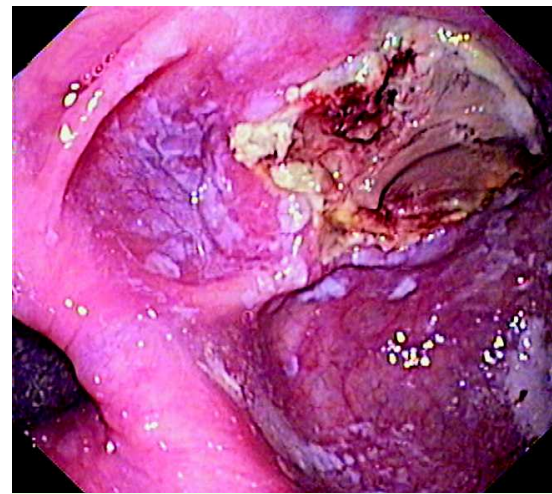

Fig. 4 Control endoscopy on day 10 , showing well-demarcated ulcer area secondary to sclerosing agent.

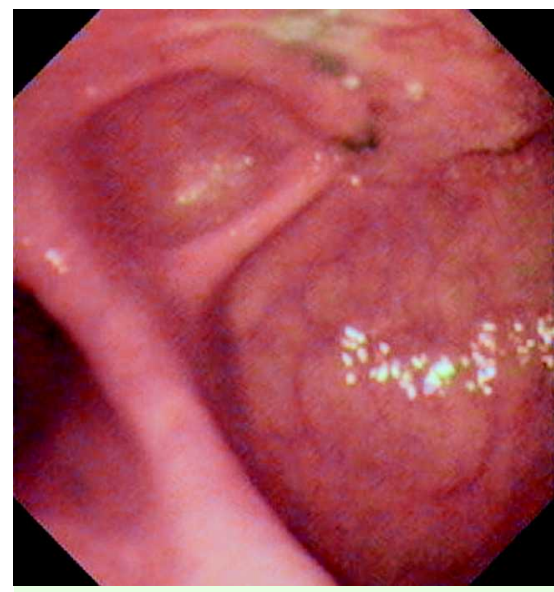

Fig. 5 Endoscopic view 1 month after the episode, showing nearly complete healing of the lesion.

A 63-year-old man presented with repeated vomiting of fresh blood. Urgent endoscopy revealed a giant midesophageal diverticulum at $28 \mathrm{~cm}$ from the incisor teeth, within which active bleeding from a protruding vessel without surrounding ulceration was seen ( $\bullet$ Fig. 1 ). The endoscopic features were compatible with a Dieulafoy's lesion. Endoscopic injection therapy was performed with epinephrine (1:10 000 dilution) and polidocanol (1\%). However, hemostasis was not achieved and spurting arterial bleeding started (๑ Fig. 2). Three hemoclips were immediately applied directly to the vessel, despite which bleeding could not be controlled. The procedure was terminated because of the high risk of esophageal perforation.
Angiographic embolization and surgery were precluded. The bleeding could be controlled with insertion of a Sengstaken-Blakemore tube. The thorax computed tomography scan revealed a huge midesophageal diverticulum but no evidence of an aortoesophageal fistula ( $\bullet$ Fig. 3). A follow-up esophagogastroduodenoscopy on day 10 revealed a well-demarcated ulcer covered by a whitish-gray exudate in the location of the previous bleeding point ( Fig. 4). This ulcer was considered secondary to the application of sclerosing agent. The last endoscopic follow-up, performed 1 month later, showed nearly complete healing of the lesion within the diverticulum ( $\bullet$ Fig.5).

Esophageal Dieulafoy's lesion is very rare and the most common site is the distal esophagus [1]. The use of the Sengstaken-Blakemore tube to control massive hemorrhage from a Dieulafoy's lesion in the lower esophagus has been described previously [2]. To our knowledge, this represents the first patient with bleeding Dieulafoy's lesion in a midesophageal diverticulum successfully managed with a Sengstaken-Blakemore tube as a rescue therapy in a patient after failed endoscopic intervention.

\section{Acknowledgment} $\nabla$

We are indebted to Dr Paul W. Bishop for his review of this manuscript.

Endoscopy_UCTN_Code_CCL_1AB_2AC_3AG

\section{Turan, A. Ozturk, U. Akarca,}

\section{O. Ozutemiz}

Section of Gastroenterology, Ege University School of Medicine, Izmir, Turkey

\section{References}

1 Lee YT, Walmsley RS, Leong RW et al. Dieulafoy's lesion. Gastrointest Endosc 2003; 58: 236-243

2 Ho KM. Use of Sengstaken-Blakemore tube to stop massive upper gastrointestinal bleeding from Dieulafoy's lesion in the lower oesophagus. Anaesth Intensive Care 2004; 32 : $711-714$

\section{Bibliography}

DOI $10.1055 / s-2007-995807$

Endoscopy 2008; 40: E177

(c) Georg Thieme Verlag KG Stuttgart · New York . ISSN 0013-726X

\section{Corresponding author}

\section{Turan, MD}

Ege Universitesi Tip Fakultesi, Gastroenteroloji Bilim Dali, Bornova 35100, Izmir, Turkey

Fax: +90-232-3731547

ilkerturan@gmail.com 